\title{
Foliar application of 5-aminolevulinic acid alleviated high temperature and drought stresses on wheat plants at seedling stage
}

\author{
Mohamed Suliman Eltyeb Suliman ${ }^{1,2}$, Safiya Babiker Mustafa Elradi ${ }^{1,2}$, Nimir Eltyb Ahmed Nimir ${ }^{1,2}$, \\ Guisheng Zhou ${ }^{1,3^{*}}$, Guanglong Zhu, ${ }^{1,3}$, Muhi Eldeen Hussien Ibrahim ${ }^{1,4}$, and Adam Yousif Adam Ali, \\ 'Yangzhou University, Joint International Research Laboratory of Agriculture and Agri-Product Safety of the Ministry of Education of \\ China, Yangzhou 225009, China. \\ ${ }^{2}$ University of Khartoum, Faculty of Agriculture, 11115 Khartoum, Sudan. \\ ${ }^{3}$ Yangzhou University, Jiangsu Co-Innovation Center for Modern Production Technology of Grain Crops, Yangzhou 225009, China. \\ *Corresponding author (gszhou@yzu.edu.cn). \\ ${ }^{4}$ Sudan University of Science and Technology, Department of Agronomy, College of Agricultural Studies, Khartoum 13311, Sudan. \\ ${ }_{5}^{5}$ University of Al Qadarif, Department of Agronomy, College of Agricultural and Environment Science, Al Qadarif 32214, Sudan.
}

Received: 22 November 2020; Accepted: 10 March 2021; doi:10.4067/S0718-58392021000300291

\begin{abstract}
Wheat (Triticum aestivum L.) seedling establishment is very sensitive to temperature and drought stresses. The present study was conducted to investigate the effects of foliar exogenous 5-aminolevulinic acid (5-ALA) application on wheat seedling under different temperature $\left(25,30\right.$, and $35^{\circ} \mathrm{C}$ ) and water regimes (normal and $65 \%$ watering). Five different levels of 5-ALA $\left(0,25,50,100\right.$ and $\left.150 \mathrm{mg} \mathrm{L}^{-1}\right)$ were applied as foliar application. Leaf area, stem diameter, shoot and root lengths, fresh and dry weights of shoot and root, and physiological parameters were measured. Drought stress $\left(\mathrm{W}_{2}\right)$ decreased leaf area by $14.9 \%$ compared with normal watering $\left(\mathrm{W}_{1}\right)$. High temperature $\left(35^{\circ} \mathrm{C}\right)$ increased malondialdehyde (MDA) content by $72.1 \%$ compared with control $\left(25^{\circ} \mathrm{C}\right) .35^{\circ} \mathrm{C}$ with $\mathrm{W}_{2}$ reduced root and shoot dry weights by $71.8 \%$ and $51.9 \%$ relative to $25^{\circ} \mathrm{C}$ and $\mathrm{W}_{1}$. At $35^{\circ} \mathrm{C}, 25 \mathrm{mg} \mathrm{L}^{-1}$ 5-ALA increased root length by $17.1 \%$ and $3.4 \%$ at $\mathrm{W}_{1}$ and $\mathrm{W}_{2}$, respectively. $150 \mathrm{mg} \mathrm{L}^{-1} 5$-ALA increased root fresh weight by $101.0 \%$ compared with $0 \mathrm{mg} \mathrm{L}^{-1} 5$-ALA at $\mathrm{W}_{1}$ and $35^{\circ} \mathrm{C}$. The highest protein content $\left(8.35 \mathrm{mg} \mathrm{g}^{-1} \mathrm{FW}\right)$ was achieved at the interaction between $\mathrm{W}_{2}, 35{ }^{\circ} \mathrm{C}$ and $50 \mathrm{mg} \mathrm{L}^{-1} 5$-ALA. At $\mathrm{W}_{2}$ and $35^{\circ} \mathrm{C}, 150 \mathrm{mg} \mathrm{L}^{-1} 5$-ALA increased proline content by $66.5 \%$ relative to $0 \mathrm{mg} \mathrm{L}^{-1}$. The results of the present study suggest that foliar application of 5-ALA could be useful to alleviate the temperature and/or drought stresses of wheat seedling.
\end{abstract}

Key words: 5-Aminolevulinic acid, crop establishment, high temperature, Triticum aestivum, water regime.

\section{INTRODUCTION}

Wheat (Triticum aestivum L.) is the third largest crop in production in the world (Fuentealba-Sandoval et al., 2020). It is critical to increase wheat production to meet the food security (Sharma et al., 2015). However, wheat growth can be affected by drought and temperature stress at any developmental stage (Pavia et al., 2019).

The temperature is predicted to rise by $2{ }^{\circ} \mathrm{C}$ by the end of this century (Zandalinas et al., 2018). High temperature can harm all the stages of plant growth from germination to reproduction, causing adverse changes in plant growth, development, physiological processes, and yield (Hussain et al., 2019). Under heat stress, wheat growth is retarded due to reduced cell division and elongation as well as decreased shoot length, root number and diameter (Iqbal et al., 2019). Heat stress can also generate excessive reactive oxygen species (ROS) including superoxide radical $\left(\mathrm{O}^{2-}\right)$ and hydrogen 
peroxide $\left(\mathrm{H}_{2} \mathrm{O}_{2}\right)$, rise lipid peroxidation, and cause deterioration in cell membrane (Djanaguiraman et al., 2018), which inevitably leads to oxidative stress (Akter and Islam, 2017).

Drought is another major stress that occurs widely and affects crop growth, development, and productivity in the world, particularly in arid and semi-arid regions (Hussain et al., 2019). All the growth and development stages are affected under drought stress by loss of turgor and subsequently disordered enzyme activities, impairment of water and nutrient uptake and transport, and decreased energy supply from photosynthesis (Ahmad et al., 2018). Moreover, drought significantly affects growth, osmotic substances, photosynthetic rate, and other physiochemical processes (Lv et al., 2020). Drought can also result increased levels of ROS in leaves.

Seedling stage is the most vulnerable phase in the life cycle of wheat plants, which is significantly reduced by abiotic stress. Seedling growth is significantly reduced by heat and drought stresses at initial stages of growth in most field crops (Yan, 2015; Ali et al., 2019). The first significant step for crop production is the successful seedling establishment, which determines the success or failure of future harvest (Finch-Savage and Bassel, 2016). Environmental stresses during initial stages are likely affect later stages of seed development and consequently grain size (Li et al., 2018).

To increase plant resistance against adverse environmental conditions, numerous substances such as plant hormones, steroids, and vitamins, have been applied exogenously to plants via seed soaking and/or foliar spraying. These compounds may improve the plants' capability to make osmotic adjustments, strengthen antioxidant enzyme defense mechanism, upregulate the biosynthesis of chlorophyll, improve radiation use efficiency, and ultimately enhance growth and biomass accumulation (Lv et al., 2020). It is reported that 5-aminolevulinic acid (5-ALA) successfully regulates the antioxidant enzyme activities and thereby increases the resistance of plants to different stresses, including chromium toxicity in sunflower, low temperature and weak light in cucumber seedling, osmotic stress in strawberry and drought stress in wheat (Anwar et al., 2020; Cai et al., 2020; Farid et al., 2020; Wang et al., 2020). The application of 5-ALA enhanced the resistance of wheat plants to drought and increased grain yield by improving growth, chlorophyll $a$ and $b$, glycine betaine, root, leaf and root $\mathrm{N}$ contents in wheat at different water regimes (Kosar et al., 2015). In addition, 5-ALA increased the growth of cucumber seedling by repressing heat stress-induced levels of malondialdehyde (MDA), $\mathrm{O}^{2-}$, and $\mathrm{H}_{2} \mathrm{O}_{2}$, and increased chlorophyll content and photosynthetic capacity (Anwar et al., 2020).

As can been from these previous studies, 5-ALA was applied only under an individual stress. There is little knowledge available on the effects of 5-ALA under combined stress of high temperature and drought. The details of the physiological and metabolic mechanisms of 5-ALA in wheat in high temperature and drought stresses still need to be elucidated. With this in mind, this study was intended to elucidate the effects of exogenous 5-ALA on morphological (root and shoot growth) and physiological measurements in wheat seedlings exposed to high temperature and drought stresses.

\section{MATERIALS AND METHODS}

A controlled pot study was done in 2019 at the Joint International Research Laboratory of Agriculture and Agri-Product Safety of the Ministry of Education of China, Yangzhou University, Yangzhou $\left(32.30^{\circ} \mathrm{N}, 119.43^{\circ} \mathrm{E}\right)$. The seeds of wheat (Triticum aestivum L.) 'Emam' were obtained from the Ministry of Agriculture of Sudan. Seeds with uniform size were sown in plastic pots $(9.5 \mathrm{~cm}$ in diameter, $8.5 \mathrm{~cm}$ in depth, and without holes at the bottom) filled with $400 \mathrm{~g}$ washed and sterilized sands. The study was designed as a 3 -factorial experiment arranged in a completely randomized design with three replicates for each treatment. Ten seeds were sown at $1 \mathrm{~cm}$ depth in each pot after full irrigation with distilled water. After 7 d, pots were separated into three growth chambers (PYX-300G-B, Yangzhou Yiwei Automatic Instrument, Jiangsu, China) setting at 25,30 , and $35^{\circ} \mathrm{C}$. All the chambers were set at $55 \%-60 \% \mathrm{RH}$ and a photoactive radiation of 500 $\mathrm{W} \mathrm{m}{ }^{-2}\left(12 / 12 \mathrm{~h}\right.$ day/night). On the $7^{\text {th }}$ day after sowing, two water regimes, i.e., normal watering $\left(80 \mathrm{~mL} \mathrm{pot}^{-1}\right)$ and drought (65\% normal watering), designated as $\mathrm{W}_{1}$ and $\mathrm{W}_{2}$ respectively, were applied at a 3-d interval based on a preliminary test on soil field capacity. Five levels of 5-aminolevulinic acid (5-ALA), 0, 25, 50, 100, and $150 \mathrm{mg} \mathrm{L}^{-1}$, were applied at a 3-d interval as foliar spray (until leaves dripped) to the seedlings on the $6^{\text {th }}$ day after seed sowing. During the study period, 100 $\mathrm{mL}$ full strength of Hoagland solution was added into each pot on the $3^{\text {rd }}, 7^{\text {th }}$, and $14^{\text {th }}$ day after seed sowing.

\section{Growth attributes}

On the $21^{\text {st }}$ day after sowing, all the seedlings were harvested. Five seedlings from each pot were randomly selected to measure stem diameter, leaf area, length, fresh weight, and dry weight of shoot and root. Stem diameter was determined 
using a vernier calliper. Lengths of shoot and root were measured using a ruler. Leaf area was calculated using the following equation (Quarrie and Jones, 1979):

Leaf area $\left(\mathrm{cm}^{2}\right)=$ Length $(\mathrm{cm}) \times$ Width $(\mathrm{cm}) \times 0.75$

The shoot and root of three plants per pot were sampled, weighed and dried in an oven at $70{ }^{\circ} \mathrm{C}$ for $3 \mathrm{~d}$ to constant weight for dry weight determination. The SPAD readings were recorded using a chlorophyll meter (SPAD-502Plus, Konica Minolta, Tokyo, Japan) at the tip, middle, and base of each leaf. The average of SPAD readings of the leaves of each pot was calculated.

\section{Physiological measurements}

The leaves of seedlings from each treatment were harvested and carefully washed with tap water, immersed in liquid nitrogen for $20 \mathrm{~min}$, and stored in a low-temperature freezer $\left(-80^{\circ} \mathrm{C}\right)$ for the determination of contents of malondialdehyde (MDA), proline and soluble protein. The content of soluble protein was determined using bovine serum albumin as the protein standard (Bradford, 1976). The proline content was determined in leaf using the method of Bates et al. (1973). The content of MDA was determined according to the method of Zhang et al. (2007).

\section{Statistical analyses}

This study was a 3-factorial design arranged in a completely randomized design with three replicates for each treatment. The data collected were subjected to ANOVA with the statistical package of MSTAT-C (Gomez and Gomez, 1984). When F values were significant, means were separated by the Tukey's test at the 0.05 probability level.

\section{RESULTS}

\section{Growth attributes}

Water regime, temperature, 5-ALA, and their interactions affected root length (Table 1). Root length was reduced with increased temperature level. At $\mathrm{W}_{1}$ and $25^{\circ} \mathrm{C}, 150 \mathrm{mg} \mathrm{L}^{-1}$ 5-ALA had the longest root length $(17.13 \mathrm{~cm})$. Moreover, 150 $\mathrm{mg} \mathrm{L}^{-1} 5$-ALA achieved the highest root length at $30^{\circ} \mathrm{C}$ with $\mathrm{W}_{2}$. At high temperature $\left(35^{\circ} \mathrm{C}\right), 25 \mathrm{mg} \mathrm{L}^{-1} 5$-ALA increased root length by $17.1 \%$ and $3.4 \%$ at $\mathrm{W}_{1}$ and $\mathrm{W}_{2}$, respectively (Table 1 ).

Water regime, temperature, 5-ALA, and their interactions significantly affected root fresh weight (Table 2). At $\mathrm{W}_{1}$ and $25^{\circ} \mathrm{C}, 100 \mathrm{mg} \mathrm{L}^{-1} 5$-ALA had the maximum root fresh weight $(1.03 \mathrm{~g})$ relative to $0 \mathrm{mg} \mathrm{L}^{-1}$ 5-ALA. Root fresh weight was increased $101 \%$ by $150 \mathrm{mg} \mathrm{L}^{-1} 5$-ALA compared with $0 \mathrm{mg} \mathrm{L}^{-1} 5$-ALA at $\mathrm{W}_{1}$ and $35^{\circ} \mathrm{C}$ (Table 1). In addition, 150 $\mathrm{mg} \mathrm{L}^{-1} 5$-ALA increased root fresh weight by $14.0 \%$ compared with $0 \mathrm{mg} \mathrm{L}^{-1} 5$-ALA at $\mathrm{W}_{2}$ and $25^{\circ} \mathrm{C}$. Water regime, temperature, 5-ALA, and the interaction between water regime and temperature significantly affected root dry weight (Table 2). Root dry weight was reduced $71.8 \%$ by $35^{\circ} \mathrm{C}$ with $\mathrm{W}_{2}$ relative to $25{ }^{\circ} \mathrm{C}$ and $\mathrm{W}_{1}$ (Table 3 ). Among different 5-ALA concentrations, the maximum root dry weight $(0.106 \mathrm{~g})$ was recorded at 0 and $25 \mathrm{mg} \mathrm{L}^{-1} 5$-ALA (Table 4).

$\mathrm{W}_{2}$ shortened shoot length by $12.3 \%$ compared with the $\mathrm{W} 1$ (Figure 1a). High temperature treatment $\left(35^{\circ} \mathrm{C}\right)$ decreased shoot length from $30.4 \mathrm{~cm}$ at $25{ }^{\circ} \mathrm{C}$ to $22.2 \mathrm{~cm}$ at $35^{\circ} \mathrm{C}$ (Table 4). Shoot length was significantly higher at $25 \mathrm{mg} \mathrm{L}^{-1}$ 5-ALA relative to other 5-ALA concentrations (Table 5). Stem diameter declined by $8 \%$ at $\mathrm{W}_{2}$ relative to $\mathrm{W}_{1}$ (Figure $1 \mathrm{~b}$ ). Stem diameter gradually decreased with increasing temperature. At high temperature $\left(35^{\circ} \mathrm{C}\right)$, stem diameter was reduced by $10.8 \%$ compared to $25^{\circ} \mathrm{C}$ (Table 4 ).

Leaf area was affected significantly by water regime, temperature and 5-ALA application (Table 2). $\mathrm{W}_{2}$ decreased leaf area by $14.9 \%$ compared with $\mathrm{W}_{1}$ (Figure $1 \mathrm{c}$ ). At 30 and $35^{\circ} \mathrm{C}$, leaf area was reduced by $16.3 \%$ and $41.3 \%$, respectively, compared with $25^{\circ} \mathrm{C}$ (Table 4). The application of $25 \mathrm{mg} \mathrm{L}^{-1} 5$-ALA had highest leaf area, although at 100 and $150 \mathrm{mg} \mathrm{L}^{-1}$ leaf area was slightly decreased (Table 5). In the interaction between water regime and temperature, the greatest reading of SPAD (36.5) was recorded in $\mathrm{W}_{2}$ and $25^{\circ} \mathrm{C}$, while the lowest (29.6) was recorded in $\mathrm{W}_{2}$ and $35^{\circ} \mathrm{C}$ (Table 3).

Shoot fresh and dry weights were significantly affected by the interaction between temperature and 5-ALA. The interaction between water regime and temperature significantly affected on shoot dry weight (Table 2 ). $\mathrm{W}_{2}$ decreased shoot fresh weight by $22.0 \%$ as compared with $\mathrm{W}_{1}$ (Figure $1 \mathrm{~d}$ ). At $30^{\circ} \mathrm{C}$, shoot fresh and dry weights were gradually reduced by increasing 5-ALA rate. At high temperature $\left(35^{\circ} \mathrm{C}\right), 150 \mathrm{mg} \mathrm{L}^{-1} 5$-ALA increased shoot fresh weight by $13.2 \%$ compared with $0 \mathrm{mg} \mathrm{L}^{-1}$ (Figure 2a). In addition, $25 \mathrm{mg} \mathrm{L}^{-1} 5$-ALA had maximum shoot dry weight $(0.26$ and $0.150 \mathrm{~g})$ at 25 and $35^{\circ} \mathrm{C}$, respectively (Figure $2 \mathrm{~b}$ ). High temperature $\left(35^{\circ} \mathrm{C}\right.$ ) and $\mathrm{W}_{2}$ reduced shoot dry weight by $51.9 \%$ compared with $\mathrm{W}_{1}$ at $25^{\circ} \mathrm{C}$ (Table 3 ). 
Table 1. Effect of the interaction between water regime, temperature, and 5-aminolevulinic acid (5-ALA) on root length, root fresh weight, and the contents of protein and proline of wheat seedlings.

\begin{tabular}{|c|c|c|c|c|c|c|}
\hline Water regime & Temperature & 5-ALA & Root length & $\begin{array}{l}\text { Root fresh } \\
\text { weight }\end{array}$ & Protein & Proline \\
\hline & ${ }^{\circ} \mathrm{C}$ & $\mathrm{mg} \mathrm{L}^{-1}$ & $\mathrm{~cm}$ & g 3 plants $^{-1}$ & $-\mathrm{m}$ & $\mathrm{FW}$ \\
\hline \multirow[t]{15}{*}{$\mathrm{W}_{1}$} & 25 & 0 & $16.81 \mathrm{ab}$ & $0.996 a$ & 7.94ij & 38.49jkl \\
\hline & & 25 & $16.06 \mathrm{abc}$ & $0.916 \mathrm{ab}$ & $8.27 \mathrm{abc}$ & $19.211 \mathrm{~m}$ \\
\hline & & 50 & $15.67 \mathrm{bcd}$ & $0.919 \mathrm{ab}$ & 7.97hij & $25.32 \mathrm{klm}$ \\
\hline & & 100 & $16.89 \mathrm{ab}$ & $1.034 \mathrm{a}$ & $8.12 \mathrm{defg}$ & 36.69jkl \\
\hline & & 150 & $17.13 \mathrm{a}$ & $0.686 \mathrm{c}$ & $7.91 \mathrm{j}$ & $41.30 \mathrm{ijk}$ \\
\hline & 30 & 0 & $16.89 \mathrm{ab}$ & $0.531 d$ & $8.21 \mathrm{bcd}$ & $35.82 \mathrm{jkl}$ \\
\hline & & 25 & $15.26 \mathrm{~cd}$ & $0.391 \mathrm{ef}$ & $8.04 \mathrm{ghi}$ & $42.52 \mathrm{ijk}$ \\
\hline & & 50 & $14.73 \mathrm{de}$ & 0.250 hijkl & $8.21 \mathrm{bcd}$ & $11.31 \mathrm{~m}$ \\
\hline & & 100 & $13.73 \mathrm{ef}$ & 0.265 ghijk & $8.21 \mathrm{bcd}$ & $11.75 \mathrm{~m}$ \\
\hline & & 150 & 11.98ghij & 0.251 hijkl & $8.17 \mathrm{bcdef}$ & $12.03 \mathrm{~m}$ \\
\hline & 35 & 0 & $11.15 \mathrm{jkl}$ & $0.172 \mathrm{jkl}$ & 8.24abcd & 53.57hij \\
\hline & & 25 & $13.06 \mathrm{fgh}$ & $0.164 \mathrm{jk} 1$ & $8.28 \mathrm{ab}$ & $61.33 \mathrm{ghi}$ \\
\hline & & 50 & $10.161 \mathrm{~m}$ & $0.159 \mathrm{jkl}$ & 8.19 bcde & $82.14 \mathrm{efg}$ \\
\hline & & 100 & 11.90hij & 0.227 hijkl & 8.08efgh & $100.72 \mathrm{cde}$ \\
\hline & & 150 & $9.85 \mathrm{~m}$ & $0.346 \mathrm{efgh}$ & $8.24 \mathrm{abcd}$ & $94.01 \mathrm{de}$ \\
\hline \multirow[t]{15}{*}{$\mathrm{W}_{2}$} & 25 & 0 & $15.83 \mathrm{bcd}$ & $0.693 c$ & $8.26 \mathrm{abc}$ & $98.47 \mathrm{cde}$ \\
\hline & & 25 & $13.90 \mathrm{ef}$ & $0.802 b c$ & $7.89 \mathrm{j}$ & $107.03 \mathrm{bcd}$ \\
\hline & & 50 & $15.84 \mathrm{bcd}$ & $0.760 \mathrm{c}$ & $8.20 \mathrm{bcde}$ & $173.00 \mathrm{a}$ \\
\hline & & 100 & $11.78 \mathrm{ij}$ & $0.465 \mathrm{de}$ & $8.22 \mathrm{abcd}$ & $97.81 \mathrm{cde}$ \\
\hline & & 150 & 12.94fghi & $0.790 \mathrm{c}$ & $8.14 \mathrm{cdefg}$ & $84.29 \mathrm{ef}$ \\
\hline & 30 & 0 & $13.44 \mathrm{f}$ & $0.380 \mathrm{efg}$ & $8.22 \mathrm{bcd}$ & $20.021 \mathrm{~m}$ \\
\hline & & 25 & $13.22 \mathrm{fg}$ & 0.304 fghi & $8.25 \mathrm{abcd}$ & $26.27 \mathrm{klm}$ \\
\hline & & 50 & $13.57 \mathrm{ef}$ & 0.279 fghij & $8.21 \mathrm{bcd}$ & $91.45 \mathrm{de}$ \\
\hline & & 100 & $13.53 \mathrm{ef}$ & $0.335 \mathrm{fgh}$ & 8.05fghi & $64.43 \mathrm{fgh}$ \\
\hline & & 150 & $13.68 \mathrm{ef}$ & 0.312 fghi & $7.85 \mathrm{j}$ & 44.89hijk \\
\hline & 35 & 0 & $11.29 \mathrm{jkl}$ & $0.199 \mathrm{ijkl}$ & $8.29 \mathrm{ab}$ & $96.80 \mathrm{de}$ \\
\hline & & 25 & $11.67 \mathrm{jk}$ & $0.166 \mathrm{jk} 1$ & $8.21 \mathrm{bcd}$ & $123.01 \mathrm{~b}$ \\
\hline & & 50 & $11.23 \mathrm{jkl}$ & 0.1341 & $8.35 \mathrm{a}$ & $118.05 \mathrm{bc}$ \\
\hline & & 100 & $11.47 \mathrm{jk}$ & $0.176 \mathrm{jkl}$ & 8.19 bcde & $157.31 \mathrm{a}$ \\
\hline & & 150 & $10.41 \mathrm{klm}$ & $0.150 \mathrm{kl}$ & $8.23 \mathrm{abcd}$ & $161.28 \mathrm{a}$ \\
\hline
\end{tabular}

Means followed by different letters in the same column are significantly different at the 0.05 probability level.

$\mathrm{W}_{1}$ : Normal watering; $\mathrm{W}_{2}$ : $65 \%$ of normal watering.

Table 2. ANOVA results for growth attributes of wheat plants as influenced by water regime, temperature, and 5-aminolevulinic acid (5-ALA) application.

\begin{tabular}{|c|c|c|c|c|c|c|c|c|c|c|c|c|c|c|c|c|}
\hline \multirow[b]{2}{*}{$\begin{array}{l}\text { Source of } \\
\text { variation }\end{array}$} & \multicolumn{2}{|c|}{$\begin{array}{l}\text { Root } \\
\text { length }\end{array}$} & \multicolumn{2}{|c|}{$\begin{array}{l}\text { Root fresh } \\
\text { weight }\end{array}$} & \multicolumn{2}{|c|}{$\begin{array}{l}\text { Root dry } \\
\text { weight }\end{array}$} & \multicolumn{2}{|c|}{$\begin{array}{l}\text { Shoot } \\
\text { length }\end{array}$} & \multicolumn{2}{|c|}{$\begin{array}{c}\text { Stem } \\
\text { diameter }\end{array}$} & \multicolumn{2}{|c|}{$\begin{array}{l}\text { Leaf } \\
\text { area }\end{array}$} & \multicolumn{2}{|c|}{$\begin{array}{l}\text { Shoot fresh } \\
\text { weight }\end{array}$} & \multicolumn{2}{|c|}{$\begin{array}{l}\text { Shoot dry } \\
\text { weight }\end{array}$} \\
\hline & MS & $\begin{array}{c}\mathrm{F} \\
\text { value }\end{array}$ & MS & $\begin{array}{c}\mathrm{F} \\
\text { value }\end{array}$ & MS & $\begin{array}{c}\mathrm{F} \\
\text { value }\end{array}$ & MS & $\begin{array}{c}\mathrm{F} \\
\text { value }\end{array}$ & MS & $\begin{array}{c}\mathrm{F} \\
\text { value }\end{array}$ & MS & $\begin{array}{c}\mathrm{F} \\
\text { value }\end{array}$ & MS & $\begin{array}{c}\mathrm{F} \\
\text { value }\end{array}$ & MS & $\begin{array}{c}\mathrm{F} \\
\text { value }\end{array}$ \\
\hline Drought (D) & 30.50 & $11.62 * *$ & 0.18 & $7.82 * *$ & 0.006 & $13.20 * *$ & 228.10 & $45.09^{* *}$ & 0.31 & $23.64 * *$ & 29.20 & $38.05^{* *}$ & 1.67 & $56.95^{* *}$ & 0.03 & $72.80 * *$ \\
\hline Temperature (T) & 129.00 & $49.41 * *$ & 3.14 & $132.40^{* *}$ & 0.078 & $173.6^{* *}$ & 637.86 & $126.09 * *$ & 0.26 & $20.06^{* *}$ & 99.80 & $130.30 * *$ & 3.14 & $107.20 * *$ & 0.07 & $148.40 * *$ \\
\hline 5-ALA (A) & 11.30 & $4.35^{\mathrm{ns}}$ & 0.07 & $3.35^{\mathrm{ns}}$ & 0.004 & $9.27 * *$ & 5.55 & $1.10 * *$ & 0.02 & $1.87^{\mathrm{ns}}$ & 0.41 & $0.54 * *$ & 0.07 & $2.43 * *$ & 0.004 & $9.005 * *$ \\
\hline $\mathrm{D} \times \mathrm{T}$ & 6.51 & $2.48 * *$ & 0.02 & $0.89^{* *}$ & 0.002 & $3.42 * *$ & 18.49 & $3.66^{\mathrm{ns}}$ & 0.01 & $0.16^{\mathrm{ns}}$ & 2.50 & $3.26^{\mathrm{ns}}$ & 0.09 & $3.26^{\mathrm{ns}}$ & 0.003 & $7.356^{* *}$ \\
\hline $\mathrm{D} \times \mathrm{A}$ & 3.16 & $1.21^{\mathrm{ns}}$ & 0.02 & $0.94^{\mathrm{ns}}$ & 0.001 & $1.40^{\mathrm{ns}}$ & 5.56 & $1.10^{\mathrm{ns}}$ & 0.02 & $1.32^{\mathrm{ns}}$ & 1.14 & $1.47^{\mathrm{ns}}$ & 0.02 & $0.68^{\mathrm{ns}}$ & 0.001 & $1.265^{\mathrm{ns}}$ \\
\hline $\mathrm{T} \times \mathrm{A}$ & 2.95 & $1.13^{\mathrm{ns}}$ & 0.02 & $0.91^{\mathrm{ns}}$ & 0.001 & $1.31^{\mathrm{ns}}$ & 7.12 & $1.41^{\mathrm{ns}}$ & 0.02 & $1.29^{\mathrm{ns}}$ & 1.12 & $1.45^{\mathrm{ns}}$ & 0.06 & $2.02 *$ & 0.011 & 2.882 ** \\
\hline $\mathrm{D} \times \mathrm{T} \times \mathrm{A}$ & 5.51 & $2.10 * *$ & 0.05 & $2.04 *$ & 0.001 & $1.25^{\mathrm{ns}}$ & 4.04 & $0.80^{\mathrm{ns}}$ & 0.01 & $0.87^{\mathrm{ns}}$ & 0.59 & $0.77^{\mathrm{ns}}$ & 0.02 & $0.51^{\text {ns }}$ & 0.001 & $0.796^{\mathrm{ns}}$ \\
\hline
\end{tabular}

*,**Significant at the 0.05 and 0.01 probability level, respectively; ${ }^{*}$ : nonsignificant; MS: mean square. 
Table 3. Effect of the interaction between water regime and temperature on root dry weight, shoot dry weight and SPAD of wheat.

\begin{tabular}{lcccc}
\hline Water regime & Temperature & Root dry weight & Shoot dry weight & SPAD \\
\hline \multirow{3}{*}{$\mathrm{W}_{1}$} & ${ }^{\circ} \mathrm{C}$ & $\mathrm{g} \mathrm{3}$ plants $^{-1}$ & $\mathrm{~g} \mathrm{3}$ plants & \\
& 25 & $0.174 \mathrm{a}$ & $0.27 \mathrm{a}$ & $34.77 \mathrm{ab}$ \\
& 30 & $0.082 \mathrm{c}$ & $0.19 \mathrm{c}$ & $32.66 \mathrm{ab}$ \\
$\mathrm{W}_{2}$ & 35 & $0.057 \mathrm{~d}$ & $0.16 \mathrm{~d}$ & $35.15 \mathrm{ab}$ \\
& 25 & $0.131 \mathrm{~b}$ & $0.21 \mathrm{~b}$ & $36.49 \mathrm{a}$ \\
& 30 & $0.084 \mathrm{c}$ & $0.17 \mathrm{~d}$ & $33.44 \mathrm{ab}$ \\
& 35 & $0.049 \mathrm{~d}$ & $0.13 \mathrm{e}$ & $29.64 \mathrm{~b}$ \\
\hline
\end{tabular}

$\mathrm{W}_{1}$ : Normal watering; $\mathrm{W}_{2}: 65 \%$ of normal watering.

Means followed by different letters in the same column are significantly different at the 0.05 probability level.

Table 4. Effect of temperature on shoot length, stem diameter, leaf area and malondialdehyde (MDA) of wheat.

\begin{tabular}{lcccc}
\hline Temperature & Shoot length & Stem diameter & Leaf area & MDA \\
\hline${ }^{\circ} \mathrm{C}$ & $\mathrm{cm}$ & $\mathrm{mm}$ & $\mathrm{cm}^{2}$ & $\mu \mathrm{mol} \mathrm{g}^{-1} \mathrm{FW}$ \\
25 & $30.38 \mathrm{a}$ & $1.67 \mathrm{a}$ & $8.79 \mathrm{a}$ & $3.80 \mathrm{~b}$ \\
30 & $29.97 \mathrm{a}$ & $1.53 \mathrm{~b}$ & $7.36 \mathrm{~b}$ & $3.61 \mathrm{~b}$ \\
35 & $22.20 \mathrm{~b}$ & $1.49 \mathrm{~b}$ & $5.16 \mathrm{c}$ & $6.54 \mathrm{a}$ \\
\hline
\end{tabular}

Means followed by different letters in the same column are significantly different at the 0.05 probability level.

Figure 1. Effects of water regime (W1: normal watering; W2: drought) on shoot length (a), stem diameter (b), leaf area (c) and shoot fresh weight (d) of five wheat plants.
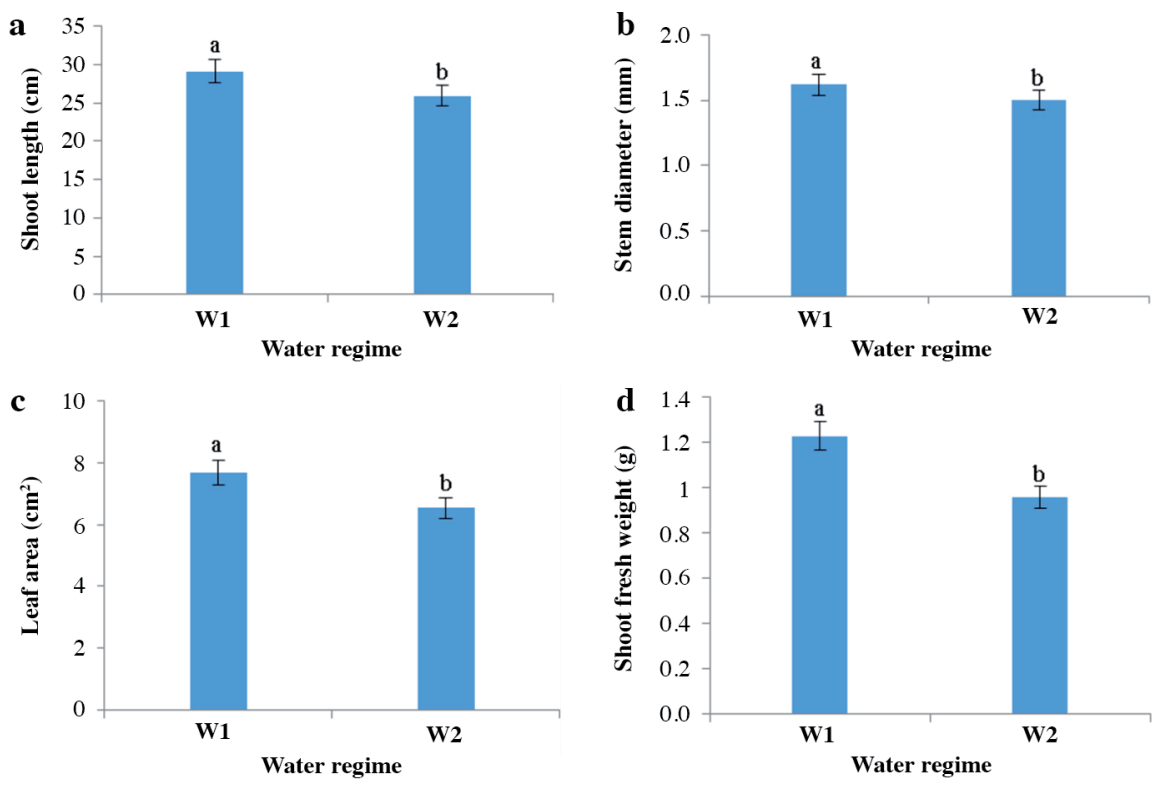

Bars with the same letters above are not significantly different at the 0.05 probability level.

Table 5. Effect of 5-aminolevulinic acid (5-ALA) on root dry weight (three plants), shoot length, and leaf area of wheat.

\begin{tabular}{cccc}
\hline 5-ALA & Root dry weight & Shoot length & Leaf area \\
\hline $\mathrm{mg} \mathrm{L}^{-1}$ & $\mathrm{~g} \mathrm{3}$ plants $^{-1}$ & $\mathrm{~cm}$ & $\mathrm{~cm}^{2}$ \\
0 & $0.106 \mathrm{a}$ & $28.52 \mathrm{ab}$ & $7.49 \mathrm{a}$ \\
25 & $0.106 \mathrm{a}$ & $28.58 \mathrm{a}$ & $7.52 \mathrm{a}$ \\
50 & $0.090 \mathrm{~b}$ & $27.05 \mathrm{bc}$ & $7.19 \mathrm{ab}$ \\
100 & $0.090 \mathrm{~b}$ & $26.24 \mathrm{c}$ & $6.78 \mathrm{~b}$ \\
150 & $0.088 \mathrm{~b}$ & $27.18 \mathrm{abc}$ & $6.65 \mathrm{~b}$ \\
\hline
\end{tabular}

Means followed by different letters in the same column are significantly different at the 0.05 probability level. 
Figure 2. Effect of the interaction between temperature and 5-aminolevulinic acid (5-ALA) on shoot fresh weight (a) and shoot dry weight (b) of three wheat plants.
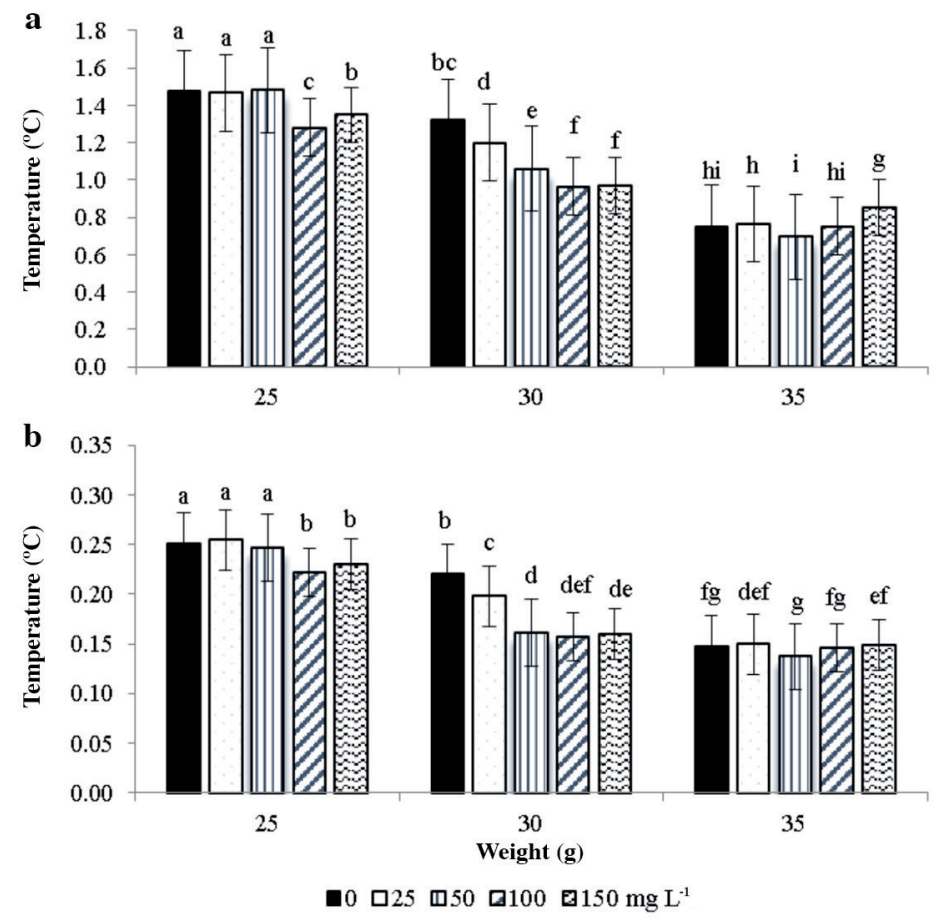

Bars with the same letters above are not significantly different at the 0.05 probability level.

\section{Physiological measurements}

Protein content was significantly influenced by the interactions between water regime, temperature and 5-ALA (Table 6). Among all the interactions, the highest protein content $\left(8.35 \mathrm{mg} \mathrm{g}^{-1} \mathrm{FW}\right)$ was achieved at the interaction between $\mathrm{W}_{2}, 35^{\circ} \mathrm{C}$ and $50 \mathrm{mg} \mathrm{L}^{-1} 5$-ALA (Table 1). Moreover, $25 \mathrm{mg} \mathrm{L}^{-1} 5$-ALA had the highest protein content at $\mathrm{W}_{1}$ with 25 and $35^{\circ} \mathrm{C}$. At $\mathrm{W}_{2}$, the highest protein content was recorded at $25 \mathrm{mg} \mathrm{L}^{-1}, 5$-ALA and $30^{\circ} \mathrm{C}$.

Water regime, temperature, 5-ALA, and their interaction significantly affected proline content (Table 6). Proline content was increased by $7.3 \mathrm{mg} \mathrm{g}^{-1} \mathrm{FW}$ at $\mathrm{W}_{1}$ and $25^{\circ} \mathrm{C}$. However, at $\mathrm{W}_{2}, 150 \mathrm{mg} \mathrm{L}^{-1}$ 5-ALA enhanced proline content by $66.5 \%$ at $35^{\circ} \mathrm{C}$ relative to $0 \mathrm{mg} \mathrm{L}^{-1} 5$-ALA (Table 1). At $\mathrm{W}_{2}, 50 \mathrm{mg} \mathrm{L}^{-1}$ increased proline content by $75.6 \%$ and $357.5 \%$ at 25 and $30{ }^{\circ} \mathrm{C}$, respectively as compared with $0 \mathrm{mg} \mathrm{L}^{-1} 5$-ALA. Moreover, $25 \mathrm{mg} \mathrm{L}^{-1}$ had the highest proline content $\left(42.5 \mathrm{mg} \mathrm{g}^{-1}\right.$ $\mathrm{FW}$ ) at $\mathrm{W}_{1}$ and $30^{\circ} \mathrm{C}$. Also, at $\mathrm{W}_{1}$ and $35^{\circ} \mathrm{C}, 100 \mathrm{mg} \mathrm{L}^{-1} 5$-ALA increased proline content by $87.9 \%$ as compared with $0 \mathrm{mg} \mathrm{L}^{-1} 5$-ALA. High temperature increased MDA content by $72.1 \%$ compared with control (Table 4 ).

Table 6. ANOVA for physiological attributes of wheat plants as influenced by water regime, temperature, and 5-aminolevulinic acid (5-ALA) application.

\begin{tabular}{|c|c|c|c|c|c|c|c|c|}
\hline \multirow[b]{2}{*}{ Source of variation } & \multicolumn{2}{|c|}{ SPAD } & \multicolumn{2}{|c|}{ Protein } & \multicolumn{2}{|c|}{ Proline } & \multicolumn{2}{|c|}{ MDA } \\
\hline & MS & F value & MS & F value & MS & F value & MS & $\mathrm{F}$ value \\
\hline Water regime (D) & 22.70 & $1.32^{\mathrm{ns}}$ & 0.02 & $0.84^{\mathrm{ns}}$ & 64156 & $89.90^{* *}$ & 0.26 & $0.04^{\mathrm{ns}}$ \\
\hline Temperature (T) & 87.55 & $5.09 *$ & 0.15 & $5.50 *$ & 35819 & $50.20^{* *}$ & 80.80 & $11.54 * *$ \\
\hline 5-ALA (A) & 116.30 & $6.78^{\text {ns }}$ & 0.04 & $1.64^{\text {ns }}$ & 5193 & $7.28 * *$ & 9.08 & $1.29^{\mathrm{ns}}$ \\
\hline $\mathrm{D} \times \mathrm{T}$ & 8.55 & $0.49 * *$ & 0.03 & $1.16^{\mathrm{ns}}$ & 2051 & $2.88^{* *}$ & 2.58 & $0.37^{\mathrm{ns}}$ \\
\hline $\mathrm{D} \times \mathrm{A}$ & 3.50 & $0.20^{\text {ns }}$ & 0.04 & $1.51^{\mathrm{ns}}$ & 2168 & $3.04 * *$ & 4.90 & $0.69^{\mathrm{ns}}$ \\
\hline $\mathrm{T} \times \mathrm{A}$ & 23.69 & $1.38^{\mathrm{ns}}$ & 0.02 & $0.86^{\mathrm{ns}}$ & 1701 & $2.19 * *$ & 1.39 & $0.19^{\mathrm{ns}}$ \\
\hline $\mathrm{D} \times \mathrm{T} \times \mathrm{A}$ & 26.42 & $1.54^{\mathrm{ns}}$ & 0.07 & $2.78 * *$ & 0.05 & $2.39 * *$ & 5.29 & $0.76^{\mathrm{ns}}$ \\
\hline
\end{tabular}

*,**Significant at the 0.05 and 0.01 probability level, respectively; ${ }^{\text {ns: }}$ nonsignificant.

MS: Mean square; MDA: malondialdehyde. 


\section{DISCUSSION}

High temperature and drought stresses are regarded as the major constraints affecting seedling growth and crop establishment. The winter season in arid and semi-arid regions (e.g., Sudan) is very short. During early seedling growth of wheat, the temperature rises quickly, resulting in poor crop establishment. The application of plant growth regulators e.g., 5-ALA at appropriate concentration could promote wheat growth in these regions and eventually leading to better crop establishment.

Stress conditions can change the visible growth attributes of the plant, which is an important indicator of stress effects. The warm environment yields lower biomass or causes dehydration related to plants grown under optimum or low temperature and subsequently restricts the growth and development of plants (Akter and Islam, 2017). Wheat seedling in this study showed reductions in leaf area, stem diameter and shoot length under high temperature (Table 4). Our results agree with those of Iqbal et al. (2019), who reported that exposure of wheat plants to heat stress reduced plant fresh weight, plant length and leaf area. Reduction in leaf size appeared an adaptive mechanism to unfavourable conditions, resulting in a decrease in photosynthesis parts and poor assimilating supply for the shoot (Ashraf and Harris, 2013; Mathur et al., 2014). Lower photosynthetic rate was detected at heat stress which leading to oxidative damage of cell organelle and thylakoid membrane lipid composition (Djanaguiraman et al., 2018). The peroxidation of unsaturated fatty acids increased MDA content, which is responsible for cell membrane disturbance (Song et al., 2016). In this study, the higher content of MDA at high temperature was observed (Table 4). The reduction in seedling growth under heat stress may be partially due to high MDA content.

Cell growth and development are the most drought-sensitive physiological processes. The growth of morphological parameters like leaf enlargement depends on turgor, temperature, and assimilates supply (Hasanuzzaman, 2020). Drought conditions lead to loss of turgor and subsequently disordered activities of enzyme, membrane injury, impairment of water and nutrient uptake, and decreased energy supply from photosynthesis (Osakabe et al., 2014). The reduction in leaf area, shoot length and stem diameter under drought stress was observed in this study (Figure 1). Our finding, similar with those of Jain et al. (2019), demonstrated that drought affects leaf size, stems extension and decreases water use efficiency. Drought-induced reduction in growth or photosynthetic rate was mostly attributed to a decreased intercellular $\mathrm{CO}_{2}$ concentration due to stomatal closure (Biswas et al., 2019).

The interaction effect of drought and high temperature stresses are usually bigger than the effects of drought or heat stress alone (Biswas et al., 2019). Our results indicate that the effect of temperature and drought together significantly reduced shoot and root dry weights (Table 3). Similarly, heat and drought stress produced substantial decreases in leaf area and DM accumulation in roots and shoots in rapeseed (Biswas et al., 2019). In these conditions, heat causes plant attempts to cool down its leaves by transpiration, while the drought has a reverse effect to avoid water loss through stomata closure (Mittler and Blumwald, 2010). Combination stress led to a higher decline in photosynthetic activity and improve production of ROS in mung bean (Nahar et al., 2017).

Use of exogenous 5-ALA increased resistance of plant to numerous abiotic stresses including drought stress (Kosar et al., 2015) and heat stress (Zhang et al., 2012). Similarly, in this study, foliar addition of 5-ALA enhanced root length, root fresh weight, under combined effects of drought and high temperature (Table 1). Our observation agrees with that of Han et al. (2018), who reported that applied 5-ALA increased fresh biomass and radicle length under drought stress.

Drought and high-temperature environments lead to osmotic stress, plants adapt to these conditions by accumulating organic or inorganic materials (Ozdeniz, 2019). Free proline and soluble protein are some of the critical organic compounds in osmotic adjustment. Application of 5-ALA promoted free proline and soluble protein contents in this study (Table 1). The accumulation of these compounds can decrease cell water potential and support to hold water of cell, relative the injury of water deficiency, and develop resistance of plants to drought stress (Liu et al., 2016). The present study is the first investigation reporting that 5-ALA application alleviates combined stress of temperature and drought at seedling stage. 


\section{CONCLUSIONS}

Our study indicated that the combined temperature and drought stress had various effects on growth and development of wheat plants. However, their effects differed considerably among various evaluated parameters. Foliar application of 5-aminolevulinic acid (5-ALA) improved root dry weight and shoot length under high temperature. In addition, 5-ALA enhanced root length, root fresh weight, protein and proline contents under combined effect of temperature and drought stresses. Exogenous 5-ALA at appropriate levels under high temperature and drought stresses conditions can be used to mitigate the deleterious effect of these abiotic stresses.

\section{ACKNOWLEDGEMENTS}

This study is financially supported by China National Key R \& D Program (2018YFE0108100) and Jiangsu Provincial Forestry Science and Technology Innovation and Extension Program (LYKJ (2019)47).

\section{REFERENCES}

Ahmad, Z., Waraich, E.A., Akhtar, S., Anjum, S., Ahmad, T., Mahboob, W., et al. 2018. Physiological responses of wheat to drought stress and its mitigation approaches. Acta Physiologiae Plantarum 40(4):80.

Akter, N., and Islam, M.R. 2017. Heat stress effects and management in wheat. A review. Agronomy for Sustainable Development 37(5):37

Ali, S., Rizwan, M., Arif, M.S., Ahmad, R., Hasanuzzaman, M., Ali, B., et al. 2019. Approaches in enhancing thermotolerance in plants: An updated review. Journal of Plant Growth Regulation 39:456-480.

Anwar, A., Wang, J., Yu, X., He, C., and Li, Y. 2020. Substrate application of 5-aminolevulinic acid enhanced low-temperature and weak-light stress tolerance in cucumber (Cucumis sativus L.) Agronomy 10(4):472.

Ashraf, M., and Harris, P.J. 2013. Photosynthesis under stressful environments: An overview. Photosynthetica 51(2):163-190.

Bates, L., Waldren, R., and Teare, L. 1973. Determination of proline for water-stress studies. Plant and Soil 39:205-207.

Biswas, D., Ma, B., and Morrison, M.J. 2019. Changes in leaf nitrogen and phosphorus content, photosynthesis, respiration, growth, and resource use efficiency of a rapeseed cultivar as affected by drought and high temperatures. Canadian Journal of Plant Science 99(4):488-498.

Bradford, N. 1976. A rapid and sensitive method for the quantitation microgram quantities of a protein isolated from red cell membranes. Analytical Biochemistry 72:248-254.

Cai, C., He, S., An, Y., and Wang, L. 2020. Exogenous 5-aminolevulinic acid improves strawberry tolerance to osmotic stress and its possible mechanisms. Physiologia Plantarum 168(4):948-962.

Djanaguiraman, M., Boyle, D., Welti, R., Jagadish, S., and Prasad, P. 2018. Decreased photosynthetic rate under high temperature in wheat is due to lipid desaturation, oxidation, acylation, and damage of organelles. BMC Plant Biology 18(1):55.

Farid, M.,Ali, S., Rizwan, M., Yasmeen, T., Arif, M.S., Riaz, M., et al. 2020. Combined effects of citric acid and 5-aminolevulinic acid in mitigating chromium toxicity in sunflower (Helianthus annuus L.) grown in Cr spiked soil. Pakistan Journal of Agricultural Sciences 57(2):477-488.

Finch-Savage, W.E., and Bassel, G.W. 2016. Seed vigour and crop establishment: extending performance beyond adaptation. Journal of Experimental Botany 67(3):567-591.

Fuentealba-Sandoval, C., Pedreros, A., Fischer, S., and López, M.D. 2020. Influence of different water deficit levels during grain filling on yield and total polyphenols content in spring wheat cultivars. Chilean Journal of Agricultural Research 80:433-443.

Gomez, K.A., and Gomez, A.A. 1984. Statistical procedures for agricultural research. John Wiley \& Sons, Hoboken, New Jersey, USA.

Han, R., Gao, G., Li, Z., Dong, Z., and Guo, Z. 2018. Effects of exogenous 5-aminolevulinic acid on seed germination of alfalfa (Medicago varia Martyn.) under drought stress. Grassland Science 64(2):100-107.

Hasanuzzaman, M. (ed.) 2020. Agronomic crops. Volume 3: Stress responses and tolerance. Springer Nature, Singapore.

Hussain, H.A., Men, S., Hussain, S., Chen, Y., Ali, S., Zhang, S., et al. 2019. Interactive effects of drought and heat stresses on morpho-physiological attributes, yield, nutrient uptake and oxidative status in maize hybrids. Scientific Reports 9(1):1-12.

Iqbal, M., Raja, N.I., Hussain, M., Ejaz, M., and Yasmeen, F. 2019. Effect of silver nanoparticles on growth of wheat under heat stress. Iranian Journal of Science and Technology, Transactions A: Science 43(2):387-395.

Jain, M., Kataria, S., Hirve, M., and Prajapati, R. 2019. Water deficit stress effects and responses in maize. in plant abiotic stress tolerance. p. 129-151. In Hasanuzzaman, M., Hakeem, K., Nahar, K., and Alharby, H. (eds.) Plant abiotic stress tolerance. Springer Nature, Cham, Switzerland. 
Kosar, F., Akram, N., and Ashraf, M. 2015. Exogenously-applied 5-aminolevulinic acid modulates some key physiological characteristics and antioxidative defense system in spring wheat (Triticum aestivum L.) seedlings under water stress. South African Journal of Botany 96:71-77.

Li, N., Xu, R., Duan, P., and Li, Y. 2018. Control of grain size in rice. Plant Reproduction 31(3):237-251.

Liu, M., Li, J., Niu, J., Wang, R., Song, J., Lv, J., et al. 2016. Interaction of drought and 5-aminolevulinic acid on growth and drought resistance of Leymus chinensis seedlings. Acta Ecologica Sinica 36(3):180-188.

Lv, J., Zong, X.-f., Shakeel Ahmad, A., Wu, X., Wu, C., Li, Y.-p., et al. 2020. Alteration in morpho-physiological attributes of Leymus chinensis (Trin.) Tzvelev by exogenous application of brassinolide under varying levels of drought stress. Chilean Journal of Agricultural Research 80(1):61-71.

Mathur, S., Agrawal, D., and Jajoo, A. 2014. Photosynthesis: response to high temperature stress. Journal of Photochemistry and Photobiology B: Biology 137:116-126.

Mittler, R., and Blumwald, E. 2010. Genetic engineering for modern agriculture: challenges and perspectives. Annual Review of Plant Biology 61:443-462.

Nahar, K., Hasanuzzaman, M., Alam, M.M., Rahman, A., Mahmud, J.-A., Suzuki, T., et al. 2017. Insights into spermine-induced combined high temperature and drought tolerance in mung bean: osmoregulation and roles of antioxidant and glyoxalase system. Protoplasma 254(1):445-460.

Osakabe, Y., Osakabe, K., Shinozaki, K., and Tran, L.S.P. 2014. Response of plants to water stress. Frontiers in Plant Science 5:86.

Ozdeniz, E. 2019. The role of free proline and soluble carbohydrates in water gypsum stress on some gypsophyte and gypsovag plants. Planta Daninha 37:e019194574.

Pavia, I., Rocha, L., Moutinho-Pereira, J., Lima-Brito, J., and Correia, C. 2019. Screening for drought resistance during germination of modern and old Iberian wheat cultivars. Acta Botanica Croatica 78(2):169-174.

Quarrie, S., and Jones, H. 1979. Genotypic variation in leaf water potential, stomatal conductance and abscisic acid concentration in spring wheat subjected to artificial drought stress. Annals of Botany 44(3):323-332.

Sharma, I., Tyagi, B., Singh, G., Venkatesh, K., and Gupta, O. 2015. Enhancing wheat production-A global perspective. Indian Journal of Agricultural Sciences 85(1):3-13.

Song, X., Wang, Y., and Lv, X. 2016. Responses of plant biomass, photosynthesis and lipid peroxidation to warming and precipitation change in two dominant species (Stipa grandis and Leymus chinensis) from North China Grasslands. Ecology and Evolution 6(6):1871-1882.

Wang, Y., Li, X., Liu, N., Wei, S., Wang, J., Qin, F., et al. 2020. The iTRAQ-based chloroplast proteomic analysis of Triticum aestivum $\mathrm{L}$. leaves subjected to drought stress and 5-aminolevulinic acid alleviation reveals several proteins involved in the protection of photosynthesis. BMC Plant Biology 20(1):1-17.

Yan, M. 2015. Seed priming stimulate germination and early seedling growth of Chinese cabbage under drought stress. South African Journal of Botany 99:88-92.

Zandalinas, S.I., Mittler, R., Balfagón, D., Arbona, V., and Gómez-Cadenas, A. 2018. Plant adaptations to the combination of drought and high temperatures. Physiologia Plantarum 162(1):2-12.

Zhang, S., Hu, J., Zhang, Y., Xie, X., and Knapp, A. 2007. Seed priming with brassinolide improves lucerne (Medicago sativa L.) seed germination and seedling growth in relation to physiological changes under salinity stress. Australian Journal of Agricultural Research 58(8):811-815.

Zhang, J., Li, D.-M., Gao, Y., Yu, B., Xia, C.-X., and Bai, J.-G. 2012. Pretreatment with 5-aminolevulinic acid mitigates heat stress of cucumber leaves. Biologia Plantarum 56(4):780-784. 